\title{
La militarisation de l'humanitaire : l'exemple somalien
}

Roland Marchal

\section{(2) OpenEdition \\ 12 Journals}

\section{Édition électronique}

URL : http://journals.openedition.org/conflits/429

DOI : $10.4000 /$ conflits.429

ISSN : $1777-5345$

Éditeur :

CCLS - Centre d'études sur les conflits lilberté et sécurité, L'Harmattan

Édition imprimée

Date de publication : 15 octobre 1993

ISSN : 1157-996X

\section{Référence électronique}

Roland Marchal, « La militarisation de l'humanitaire : l'exemple somalien », Cultures \& Conflits [En ligne], 11 | automne 1993, mis en ligne le 13 mars 2006, consulté le 30 mars 2021. URL : http:// journals.openedition.org/conflits/429; DOI : https://doi.org/10.4000/conflits.429

Ce document a été généré automatiquement le 30 mars 2021.

Creative Commons License 


\title{
La militarisation de l'humanitaire : l'exemple somalien
}

\author{
Roland Marchal
}

L'opinion publique internationale s'est émue, par journalistes interposés, du spectacle de désolation qu'offraient certaines zones de la Somalie à l'automne 1992 et de ces adolescents armés de kalashnikov aux mines farouches qui s'adonnaient, tantôt aux tâches de sécurité, tantôt au pillage le plus éhonté de l'aide d'urgence. Elle a respiré d'aise lorsque les soldats de la force internationale sont intervenus en décembre 1992 pour mettre fin aux rapines et nourrir ceux qui mourraient de faim. Cette candeur devant des images génériques de tout désastre humanitaire fait problème pour de nombreuses raisons. Pourquoi s'émouvoir et se scandaliser devant la tragédie somalienne, alors que celle-ci durait déjà depuis de très longs mois sans réaction de la communauté internationale? Pourquoi concentrer une telle attention sur quelques zones de la Somalie, alors qu'ailleurs dans le monde, à commencer par le Soudan voisin, les droits les plus élémentaires étaient bafoués mettant en péril la vie de centaines de milliers d'innocents? Pourquoi, enfin, occulter une fois de plus le rôle ambigu que joue dans toutes les situations de guerre l'aide humanitaire, qui fut, notamment dans la Corne de l'Afrique, l'une des composantes de l'économie politique des conflits ${ }^{1}$ ? La réponse à ces questions n'est certes pas immédiate, car elle exige une analyse non seulement des situations de crise, mais également des sociétés où le marché humanitaire s'est le mieux développé. Un analyste notait sur un ton désabusé à propos des interventions internationales dans les famines de la Corne de l'Afrique que "Toutes ont eu lieu entre une élection présidentielle américaine et la fin de l'année. Les semaines qui suivent l'élection ou la réélection d'un candidat à la présidentielle, "dévoué à la cause de tous et à la lutte contre la récession intérieure", alors que Thanksgiving et Noël approchent, constituent une période où la conscience politique américaine est la plus vulnérable"2. Force est de constater en tout cas qu'il n'y a aucun lien obligé entre l'intensité d'une crise et l'attention prodiguée par la communauté internationale. Cette absence de facteurs objectifs dans la réaction extérieure n'est pas la moindre aporie dans la constitution d'un droit d'ingérence humanitaire. La Somalie fournit un bon paradigme pour s'interroger sur les relations ambiguës 
qu'entretiennent le militaire et l'humanitaire. On pense immédiatement à l'intervention de décembre 1992 de l'United Task Force (UNITAF) dont le mandat, défini par la résolution 794 le Conseil de sécurité, permettait l'emploi de la force pour assurer la distribution de l'aide d'urgence aux mouroirs somaliens, et à ONUSOM II (Opération des Nations unies pour la Somalie), qui a pris la relève en mai 1993, et qui est la première opération de l'O.N.U., depuis sa fondation, à être basée sur le chapitre VII de sa charte autorisant l'utilisation de la force pour appliquer le mandat donné par la résolution 814. Mais, les péripéties de cette action et l'attitude des acteurs somaliens s'inspirent également d'un legs historique plus ancien : le régime Siyad Barre lui-même dont la chute en 1991 inaugure la crise et les deux ans qui séparent l'insurrection de Mogadiscio de l'arrivée de l'UNITAF dans la capitale somalienne.

Le régime Siyad Barre et la constitution d'une culture de l'aide Trop souvent en effet, les observateurs oublient que l'intervention humanitaire en Somalie est antérieure à la crise de la dictature. Elle a joué un rôle non négligeable dans la survie du régime autoritaire, tout en contribuant à l'élaboration d'une culture de l'aide, qui, pour n'être pas spécifique à ce pays, possède des traits propres en Somalie, traits qui n'ont pas été altérés par trois années de convulsions politiques très violentes. Fondamentalement, l'Etat somalien a su profiter au maximum de la rente de situation qu'autorisait la polarisation Est/Ouest dans la région ${ }^{3}$. Il a su également user de la rhétorique tiers-mondiste et faire valoir sa pauvreté pour demander à la communauté internationale les moyens de pallier aux conséquences des sécheresses désastreuses depuis l'indépendance, notoirement après 1970. C'est surtout après la guerre de l'Ogaden en 1978, que la Somalie a utilisé l'aide humanitaire dans une stratégie de consolidation d'un ordre politique, qui avait été mis en cause par sa défaite face à l'Ethiopie. En effet, profitant de la déliquescence de l'appareil d'Etat après la déposition d'Haïle Sellassie en octobre 1974, les périphéries de l'Ethiopie s'étaient révoltées contre le pouvoir central : durant l'été 1977, les forces du Front de libération de la Somalie occidentale, considérablement appuyées par l'armée somalienne, rentrèrent en Ogaden afin d'en expulser l'armée éthiopienne. Ce conflit donna lieu à un saisissant renversement d'alliances puisque les Soviétiques, alliés de la Somalie depuis 1963, optèrent pour le soutien à la révolution éthiopienne, alors que les Etats-Unis stoppaient leur très ancienne coopération avec Addis-Abéba et garantissaient la survie de l'Etat somalien. L'avancée victorieuse des troupes de Siyad Barre fut arrêtée grâce à l'arrivée du contingent cubain et à un pont aérien soviétique qui déversa les fournitures militaires nécessaires à la contre-offensive éthiopienne qui provoqua une véritable déroute somalienne. Dans les années qui suivirent près d'un million de réfugiés d'Ogaden entrèrent en Somalie, dans la région du nord-ouest (l'ancienne colonie anglaise), la Région centrale et le sud de la Somalie. Le régime de Siyad Barre devint donc dépendant de l'aide humanitaire octroyée par les Etats occidentaux et le Haut Commissariat aux réfugiés des Nations unies (H.C.R.) ${ }^{4}$. Confronté d'abord à une tentative de coup d'Etat dès 1978, puis à des mouvements armés, le dictateur fit de plus en plus appel à ces réfugiés, membres du clan Ogaden, pour constituer des milices acquises à son régime et combattre les rébellions dont les bases arrières étaient en Ethiopie. Cette politique lui permettait de consolider le premier cercle de sa base sociale, tout en marginalisant les clans et les régions les plus hostiles à son pouvoir ${ }^{5}$. L'aide humanitaire fut donc associée à une stratégie politique et militaire explicite du régime autoritaire. Certes, elle allait aux réfugiés mais dans des proportions de plus en plus réduites. Une audit de l'U.S.A.I.D. en 1986 affirmait que 12\% seulement de l'aide 
attribuée par cet organisme atteignait réellement les réfugiés. Le taux moyen des détournements pouvait atteindre alors jusqu'à $75 \%$. L'Etat somalien utilisait l'essentiel de ces dons pour rétribuer ses clientèles ou pour nourrir son armée et ses milices. Ce comportement n'était sans doute pas très original, puisqu'on le retrouve sous une forme à peine atténuée dans l'Ethiopie de Mengistu, lors de la famine de 1985. Ce qui est sans doute caractéristique de la Somalie, c'est que les Etats donateurs ont fermé les yeux, année après année, sur les malversations auxquelles l'aide humanitaire donnait lieu, sans jamais exiger une plus grande modération. Plusieurs représentants du H.C.R. furent expulsés pour avoir contesté les chiffres faramineux de réfugiés fournis par l'Etat somalien, sans que New-York ne s'en émeuve outre mesure. Lorsque la guerre civile s'aiguisa à la fin des années 80 , entraînant destructions massives, massacre de troupeaux et de la population civile ${ }^{7}$, non seulement, les pressions des principaux donateurs, qu'étaient les USA et l'Italie, restèrent d'une très grande modération mais les programmes d'assistance militaire ne furent stoppés que très tardivement. Malgré les bombardements sur la population civile et des violations des droits de l'homme sans précédent, l'administration Bush présenta au Congrès pour l'année fiscale 1991, un programme de formation militaire de 900.000 U.S.D., qui "aurait continué d'exposer les officiers somaliens aux valeurs américaines de respect des droits de l'homme" (sic) ${ }^{8}$. Ces quelques annotations veulent seulement indiquer que, pendant une période relativement longue, la coopération internationale et humanitaire a servi en Somalie de ressource d'appoint à une dictature sanglante et à son appareil de coercition, qui ne s'embarrassaient d'aucune conditionalité démocratique, humanitaire, encore moins de "good governance" prônée pourtant par les agences financières internationales. Les donateurs faisaient preuve d'une extrême complaisance, simplement parce que la Somalie était opposée à l'Ethiopie sous influence soviétique. L'aide était donc perçue comme une rente et un droit; questionner son emploi ou ses destinataires ne relevait pas de la compétence des donateurs. A ces considérations, qu'on peut retrouver dans d'autres situations sous une forme moins radicale, s'en ajoutait également une autre : le comportement des coopérants, leur salaire et la désinvolture avec laquelle ils réagissaient devant les détournements accréditaient socialement l'idée que l'argent ou les ressources mises à disposition par la communauté internationale n'appartenaient à personne, c'est à dire à tout le monde. De la même manière, l'idée que l'aide était destinée aux plus nécessiteux et aux populations les plus précarisées par l'exil ou la sécheresse n'avait plus rien d'évident, tant sa fonction première était la rétribution des clients du pouvoir. Cette non discrimination entre bien privé et bien public renvoie sans aucun doute à des problématiques plus complexes que les dysfonctionnements de l'aide internationale ou la prévarication propre à tout régime autoritaire : elle exige de s'interroger sur les structures sociales et politiques de la Somalie et sur la logique segmentaire considérablement polarisée par la dictature, pendant les dernières années de son existence. Reste que l'intervention humanitaire durant l'ère Siyad Barre n'aura guère contribué à dissiper les confusions mais, au contraire, aura considérablement cultivé les malentendus.

3 L'époque héroïque des ONG Pour analyser les relations entre humanitaire et militaire avant l'intervention de décembre 1992, il convient sans doute de distinguer deux périodes qui présentent de nombreux points communs mais qui diffèrent par la nature de la présence internationale et les formes d'aide. La première s'étend du début 1991 au printemps 1992 et est marquée par une très faible présence d'ONG qui, si on les considère comme des opérateurs économiques, n'ont qu'une influence relativement 
marginale ; la seconde est plus brève et correspond à la première période des Nations unies en Somalie, du printemps à l'hiver 1992, avec un déversement impressionnant d'aide d'urgence. En décembre 1990, la situation s'est grandement détériorée dans l'ensemble de la Somalie sous l'action conjuguée des différents fronts d'opposition armée $^{9}$ : Siyad Barre ne tient plus l'intérieur du pays, justifiant malgré lui le surnom dont l'affublent les insurgés "le maire de Mogadiscio". Les agences internationales et les ONG se sont repliées dans la capitale où la sécurité n'est guère brillante; leurs véhicules sont "confisqués" par la guérilla et transformés en "technical cars" ${ }^{10}$, ou, plus prosaïquement, volés par des brigands qui les revendent en Ethiopie ou au Kenya. Elles quittent toutes ${ }^{11}$ la Somalie en décembre, alors que l'insurrection couve, ou au début du mois de janvier 1991, lorsque les combats font déjà rage dans la capitale. Quelques ONG., pourtant, s'efforcent de prendre pied dans les premières semaines de 1991 dans une situation très difficile, compte tenu de la précarité de la situation militaire et de la compétition déjà marquée entre les dirigeants des deux factions de l'United Somali Congress (U.S.C.), Ali Mahdi Mohamed qui s'auto-proclame président le 27 janvier 1991 au lendemain du départ de Siyad Barre de la capitale, et le général Mohamed Farah Hassan Aydiid qui obtient en juillet 1991 la présidence de l'U.S.C. Cette compétition factionnelle dans une atmosphère très tendue est d'autant plus dangereuse que Siyad Barre n'a pas encore renoncé à tout espoir de retour : jusqu'à sa défaite définitive en mai 1992, offensives et contre-offensives déchirent le sud de la capitale, notamment la région de Bay où la famine fait des ravages dans les populations d'agriculteurs, dès l'automne 1991. Ce climat politique et militaire, pour le moins délétère, limite considérablement le travail des ONG et leur interdit une présence, même semipermanente, en dehors des grands centres urbains jusqu'au printemps 1992, malgré quelques exceptions notables dans le nord-ouest (le Somaliland) et le nord-est (Majerteeniya). Dans la capitale, la normalisation est très fragile; plusieurs incidents armés éclatent avant une première confrontation violente d'une semaine entre les deux chefs de guerre en septembre 1991, et surtout une véritable bataille rangée sans merci de novembre 1991 à mars 1992. Durant cette première période, les ONG qui désirent s'installer n'ont guère le choix dans les moyens d'assurer leur présence et leur sécurité car, malgré les déclarations des différents chefs de faction leur promettant gracieusement combattants ou "policiers", ils doivent recruter des gardes et payer leur armement. La location d'une voiture ou d'une maison implique l'emploi de gardes dont le nombre est âprement négocié avec le propriétaire, souvent notable d'un clan qui réalise ainsi une double opération : assurer des ressources à ses dépendants et gagner un pécule absolument nécessaire pour concrétiser ses ambitions politiques. Globalement, pourtant, ce système va relativement bien fonctionner. Certes, au début, le personnel médical est menacé plusieurs fois par des combattants qui ramènent un de leurs compagnons d'arme blessé et qui n'admettent pas de le voir soigné après des civils, des femmes ou des enfants ; mais ces moments de tension s'espacent rapidement. Les voitures et le personnel des ONG bénéficient durant cette période d'une relative immunité qui est l'un des résultats paradoxaux de « l'équilibre de la terreur » qui parait alors définir la situation dans la capitale : dans la mesure où leurs gardes appartiennent aux clans les plus puissants, s'attaquer aux biens des ONG revient à encourir des représailles de l'ensemble du clan. Cette observation peut d'ailleurs s'inscrire dans une constatation plus générale, à savoir que le tissu social et économique s'est recomposé dans la guerre et qu'une certaine forme d'équilibre s'est ainsi construite, avec un coût humain certes important ${ }^{12}$. Cependant, cette affirmation ne peut occulter les problèmes 
auxquels les événements vont confronter les ONG. Le plus important est celui de leur neutralité. Installées dans un quartier de la capitale, employant plusieurs dizaines, voire centaines, de Somaliens, souvent sans aucune maîtrise réelle sur leur choix, les ONG sont perçues par la population comme les financiers de tel ou tel clan, de telle ou telle faction : les déclarations de neutralité ou d'indépendance ne convainquent guère ${ }^{13}$. Cette situation n'est certes pas rédhibitoire en situation de paix, mais il en est tout autrement lorsque des affrontements éclatent. Ainsi, lors de la bataille de Mogadiscio ${ }^{14}$, l'accès aux victimes, qui constitue le fondement de l'action des ONG et est censé légitimer leur existence, n'a été possible que d'une manière très partielle à cause de ces identifications. D'une part, la ville était coupée en deux et les combats limitaient très fortement les déplacements. D'autre part, malgré toutes les assurances prodiguées par les leaders des factions armées, les convois de médicaments étaient le plus souvent stoppés à un barrage et sommés de rebrousser chemin car il était interdit de passer dans une zone qui n'était pas sous contrôle. Ce problème était d'autant plus dramatique que les hôpitaux étaient tous situés dans Mogadiscio-sud tenu par le général Aydiid. Un second problème particulièrement sérieux est le financement du conflit. On n'évoquera pas ici le détournement d'une partie de l'aide et des médicaments qui allaient fournir les dispensaires et les cliniques privées somaliennes; la corruption et l'esprit d'entreprise n'avaient pas disparu avec la dictature; cependant, ces détournements n'étaient pas très significatifs car les volumes concernés n'avaient rien de comparable avec l'époque antérieure ou avec celle qui allait suivre. Il serait faux de voir dans les ONG les principaux financiers de la guerre civile somalienne. Il n'en demeure pas moins qu'elles ont apporté leur modeste obole, bien involontairement sans aucun doute. Cette question se pose dans tous les conflits armés mais, en général, les ONG assument ce choix même s'il n'est pas porté sur la place publique: on peut le discuter mais il recouvre une dimension et un positionnement indiscutablement politique. Ceux-ci existaient par exemple en Erythrée ; était-ce le cas dans la guerre de Mogadiscio et cela justifiait-il une implication même mineure des ONG dans un tel processus guerrier? Ainsi des gardes d'ONG ont abandonné pendant plusieurs jours leur poste et sont partis avec leur armement faire le coup de feu dans la capitale, pour reprendre peu après leur fonction sans sanction d'aucune sorte. Certains responsables d'ONG ne cachent pas leur malaise devant cette situation mais ils soulignent que ces mêmes gardes ont fait preuve d'une grande intégrité dans leur travail et ont souvent pris des risques importants pour permettre aux ONG de travailler, et qu'il aurait été impensable d'interrompre leur mission à un moment où les hôpitaux regorgeaient de blessés. Reste une double interrogation sur la dimension éthique dont se pare le petit monde des ONG et la popularité que gagnaient celles qui étaient présentes durant ces heures difficiles en Somalie dans les opinions publiques occidentales et sur le marché humanitaire. La seconde période est celle de la "redécouverte" de la Somalie par la communauté internationale. Après des mois d'indifférence et de tergiversations, les Nations unies ont négocié un cessez-le-feu entre les deux belligérants de Mogadiscio, qui prit effet le 3 mars $1992^{15}$. Celui-ci préfigurait le retour des grandes agences et des ONG et le déversement tardif mais imposant d'une aide d'urgence pour tenter d'enrayer l'une des pires famines qu'aura connue la Corne de l'Afrique. Rétrospectivement, ce qui frappe le plus l'observateur est l'incroyable amnésie ou naïveté de ces nouveaux intervenants : la seule dimension prise en compte est la famine, la crise sociale et politique, ses implications militaires sont proprement exclues du champ d'analyse de la communauté internationale; cet aveuglement allait considérablement fragiliser l'ensemble de la 
démarche onusienne avant et après décembre 1992. Les ONG présentes depuis 1991 avaient acquis une expérience et démonté quelques uns des effets pervers de cette économie de guerre. Mais l'O.N.U. et ses agences, qui n'auraient pas dû être démunies devant de tels problèmes après le Liban ou le Cambodge, les ont traitées avec la plus grande désinvolture et ont reproduit à une grande échelle, avec une toute autre intensité, des erreurs qui accélèrent la crise de l'automne, d'autant que les budgets onusiens ne sont guère comparables à ceux des ONG, pas plus d'ailleurs que les conditions dans lesquelles leurs expatriés acceptent de vivre. Les ONG, nouvellement arrivées mais pressées de démontrer leur indépendance de vue, ont hélas eu la même réaction, ce qui suscita quelques bons mots de la part des Somaliens : Concern/Irlande est devenu un groupe Hawadle, A.I.C.F./France a opté pour les Sheikhal, etc. Les conséquences d'un tel aveuglement ont été rapidement perceptibles. Une partie des miliciens et de l'armement a été recyclée dans la protection des agences et des ONG. Les contracteurs ont obtenu des contrats léonins pour le transport des vivres, la location de voitures ou d'habitations, etc. Le pillage de l'aide humanitaire, de sporadique au printemps, est devenu systématique et organisé pendant l'été. Pour expliquer cette évolution qu'il faudrait plus nuancer, trois dimensions importantes dans le fonctionnement de l'aide doivent être prises en compte. Tout d'abord, faute de stratégie politique, à aucun moment la communauté internationale n'a perçu la nécessité du désarmement, quel qu'en eût été la forme, comme un élément essentiel dans les tractations avec les chefs de guerre. Aussi, les combattants qui n'ont pu trouver un emploi dans les circuits humanitaires ont souvent quelque difficulté à voir passer sous leurs yeux un tel pactole sans se servir, d'autant que cette aide est destinée à des groupes sociaux, qu'ils considèrent avec beaucoup de mépris ${ }^{16}$, et que l'absence d'expédition guerrière dispense les clans ou les chefs de faction de leur donner un petit pécule ou de leur promettre un butin. Ensuite, les dirigeants somaliens profitent de l'armistice et de la manne humanitaire pour rembourser leurs financiers, après une très longue et coûteuse confrontation, qui n'a, de plus, pas encore trouvé de conclusion définitive. Cela explique pourquoi les "taxes" ou les détournements de l'aide vont prendre une forme de plus en plus organisée et systématique avec le temps. Enfin, tout le monde estime que l'intervention onusienne préfigure la constitution d'un gouvernement national. Il s'agit donc de mener campagne afin de consolider des alliances et de recruter de nouveaux partisans, c'est à dire de dépenser de l'argent, de multiplier les cadeaux aux elders, d'envoyer quelques camions de vivres dans les bases rurales de son clan, etc. C'est donc une nouvelle fois vers l'aide humanitaire que se tourne ce petit monde. Dans toutes ces situations, les discours tenus par les acteurs somaliens sur l'aide internationale participent des mêmes a priori que dans la période Siyad Barre. Personne ne s'émeut lorsque Aydiid convoque parmi ses financiers les Somaliens qui avaient les plus hautes responsabilités dans le C.I.C.R. ; les Nations unies paient mois après mois des contracteurs dont les camions ont la fâcheuse tendance de se vider durant leur parcours ; les gens en armes sont toujours les premiers allocataires de l'aide internationale. La suite de la crise somalienne a mis entre parenthèses ces deux années, sans même évoquer le règne de Siyad Barre qui n'a sans doute jamais existé si l'on en croit les déclarations en juin 1993 du représentant spécial des Nations unies en Somalie, qui rendait responsable le seul Aydiid de la dévastation la Somalie. La rapide évocation de ces moments souligne pourtant l'absolue nécessité d'une réflexion des ONG sur leur insertion dans des conflits armés: celles-ci, considérablement échaudées par les performances pitoyables des Nations unies en Somalie, ont tendance 
à se concentrer sur leur relation très problématique avec les casques bleus et à occulter les problèmes qu'a soulevés leur présence en Somalie avant l'arrivée de l'U.N.I.T.A.F. Elles semblent vouloir croire que les mouvements armés avec lesquels elles interagissaient étaient des mouvements de libération "classiques", alors qu'ils n'étaient plus que des instruments d'entrepreneurs politico-militaires, prêts à tout pour gravir les marches du pouvoir. Dans les dernières semaines qui précèdent l'intervention de décembre, la presse internationale a mis l'accent sur les taxes que devaient payer les ONG pour travailler en Somalie ou sur le coût des gardes qu'elles louaient. Cette situation soulevait des problèmes de principe réels, auxquels ONG et communauté internationale devront apporter des réponses. Cependant, il est aussi utile de rappeler que ce fonctionnement n'est pas aussi pathologique que certains esprits moralisateurs l'ont dit. D'une part, on pourrait démontrer de façon très argumentée que le pillage a eu une incontestable fonction de redistribution sociale et de remonétisation de l'économie, tâches dans lesquelles ni les ONG, ni les agences des Nations unies n'ont vraiment brillé. Cela a incontestablement permis aux populations pastorales d'échapper à la famine, après les mauvaises pluies de 1991 et la vente de leurs troupeaux à des prix sacrifiés durant la guerre de Mogadiscio pour soutenir l'effort de guerre. D'autre part, si l'on compare les pratiques des factions somaliennes avec celles des Etats voisins, on voit que les coûts occasionnés par ces taxes sur l'aide n'ont sans doute guère été supérieurs à la moyenne, simplement les mécanismes d'extorsion ont été plus apparents. L'Ethiopie a toujours fait payer des droits de douane sur l'aide d'urgence; quant au Soudan, il a obligé pendant des années les organisations humanitaires à changer les fonds nécessaires à leurs activités à un taux près de trois fois inférieur au taux normal. Les Etats font souvent preuve de plus de flexibilité devant le moralisme des bonnes consciences occidentales...

4 De l'UNITAF à l'ONUSOM : l'échec répété On ne reviendra pas ici sur les différentes motivations du président Bush et des Nations unies qui donnent une telle dimension militaire à l'intervention de décembre ${ }^{17}$. Il faut néanmoins mettre en cause ce qui fut la perception commune, mais erronée, en novembre-décembre 1992 de la Somalie. Tout d'abord, la Somalie a été réduite aux zones mouroirs de la région de Bay; il n'y a eu aucune analyse des causes de la famine, encore moins de la situation politique qui l'expliquait en grande partie. La cause de taux de décès très élevés était claire : les bandits empêchaient l'aide d'arriver à ceux qui en avaient le plus besoin. Le Secrétaire général des Nations unies, qui n'aura guère brillé sur ce dossier, évoquait des détournements de l'aide de $80 \%$, chiffre qui sera démentie par tous les intervenants en Somalie, tant agences qu'ONG : qu'importe puisqu'il s'agissait d'entériner une décision américaine et de justifier un nouvel interventionnisme prescrit par son Agenda pour la paix $^{18}$. Ce refus d'assumer les dimensions politiques et sociales de la crise somalienne a réduit l'intervention internationale à sa plus simple expression : policière. La priorité est donc de sécuriser les routes et de permettre l'approvisionnement régulier des camps de déplacés, but honorable mais sans lendemain, s'il n'est assorti de mesures complémentaires. Les questions centrales du désarmement, de la démobilisation, de la restauration de la paix civile et d'une légalité doivent attendre plusieurs mois avant d'être réellement considérées par l'UNITAF et l'ONUSOM. Les conséquences néfastes de cet impensé apparaissent rapidement, même si l'arrivée de plus de 35000 soldats étrangers a des effets positifs qu'on ne peut ignorer : la population civile ne sent plus otage des gens en armes, l'aide humanitaire arrive dans les zones les plus affectées par la famine ${ }^{19}$. La sécurité demeure précaire en ville et disparaît dans les campagnes car de 
nombreux combattants optent alors pour le banditisme, faute d'alternative de reclassement dans une société pacifiée. Si les armes lourdes sont encore utilisées dans les campagnes, en ville ce sont les poignards et les revolvers qui deviennent les plus usités. L'équilibre de la terreur et la peur de représailles qui permettaient une certaine régulation de la violence dans la période précédente ne fonctionnent plus et les incidents deviennent plus meurtriers. ONG et Somaliens paient en quelques mois un lourd tribut à l'inconséquence de l'O.N.U. et des Etats-Unis. Les possibilités de déplacements dans les campagnes diminuent de façon drastique et exigent une escorte que les forces de la coalition internationale n'accordent pas toujours. Le nombre de blessés ou de tués dans des agressions augmente notablement. La tension entre ONG et UNITAF, puis ONUSOM, n'a donc pu que croître dans la mesure où leurs logiques d'action sont totalement différentes. Les soldats de l'UNITAF ou les casques bleus raisonnent en termes purement militaires avec, comme priorité numéro un, leur propre sécurité20 ${ }^{2}$. Les ONG s'efforcent de répondre à ce qu'elles pensent être les besoins de la population somalienne; elles privilégient donc l'accès aux groupes précarisés. L'arrivée des troupes internationales souligne une nouvelle fois l'ambiguïté des relations entre gardes et ONG. Certes, ceux-ci ont permis pendant de longs mois au personnel humanitaire de sillonner le pays et de prodiguer des soins à la population. Cependant, les ONG ont également pu être leurs "otages", dans la mesure où ils étaient seuls maîtres de l'appréciation des conditions de sécurité et menaient à l'occasion des activités parallèles particulièrement répréhensibles en utilisant les locaux de leurs employeurs comme sanctuaires. De telles prérogatives disparaissent soudainement en même temps que se profile la possibilité de licenciements. Dans plusieurs ONG, cette situation donne lieu à d'importantes tensions et à des menaces physiques, qui provoquent dans un cas au moins la mort d'un expatrié... Les relations avec les casques bleus se révèlent assez problématiques. Les priorités ne sont pas les mêmes, les modes de fonctionnement non plus. Des officiers se plaignent de voir des escortes attendre en vain des voitures d'ONG qui ont remis leur déplacement au dernier moment, ou ne comprennent pas l'utilité d'un déplacement dans une zone où celles-ci n'ont aucune activité. De l'autre coté, les récriminations ne manquent pas non plus: les ONG rappellent que les casques bleus sont là pour les protéger et qu'ils doivent se conformer à cette priorité, ce qui est, le plus souvent, loin d'être le cas. A Kismayo, un officier pris à parti par une ONG qui lui rappelait le mandat de l'UNITAF répondit qu'elle n'avait qu'à louer les services de mercenaires sud-africains... Les relations entre ONG et troupes internationales vont évoluer de la même manière que celles entre casques bleus et population somalienne. En effet, le mandat octroyé à l'UNITAF puis à l'ONUSOM donne une très grande lattitude aux soldats sur le terrain: leur comportement face aux civils ne fait pas toujours l'objet d'un contrôle suffisant, leur formation ne les aide pas non plus à assumer des tâches qui relèvent plus d'une police militaire que d'une armée. Très vite, des abus sont commis et aliènent une partie de la population ${ }^{21}$ : des Somaliens sont tués, blessés ou brutalisés sans réelle justification, des vols sont commis, etc. Les $\mathrm{ONG}$, déjà critiques à cause des multiples limitations induites par la présence des casques bleus, réagissent avec vigueur devant des faits, qui sont souvent avérés, même s'ils ne concernent qu'une fraction marginale des soldats de l'UNITAF ou de l'ONUSOM. Il serait facile de mettre cette réaction sur le compte d'un anti-militarisme propre aux jeunes générations du personnel des ONG, comme le suggèrent certains officiers américains. L'argument n'est peut-être pas faux mais les violations des droits de l'homme exercées par ceux qui se veulent les représentants de 
la légalité internationale sont bien réelles et les ONG redoutent avec raison d'être associées à de telles pratiques et de voir le climat de confiance nécessaire à leur travail disparaître. La crise qui éclate à Mogadiscio après l'assassinat de 23 casques bleus pakistanais le 5 juin 1993 sert de révélateur à tous ces problèmes, qui existaient depuis de longs mois sur un mode plus ou moins mineur. On mesure mieux alors les impasses de l'intervention internationale, son improvisation, et l'absence de stratégie politique, aspects que l'ONUSOM essaie d'occulter par des gesticulations militaires qui ont mené à l'impasse actuelle. Cette vision très rudimentaire de la crise somalienne, qui perdure depuis décembre, a provoqué une série de glissements sémantiques puis politiques dans la perception des autorités onusiennes. Le général Aydiid, coopté en décembre par les Américains, devint un chef de guerre peu recommandable au printemps et un bandit en juin : aucun de ces qualificatifs n'est contestable, aucun n'est exclusif des autres. Mais, en faisant du général Aydiid un simple délinquant, l'ONUSOM a oublié que, bien qu'affaibli et mis en cause par une partie de ses partisans, il est toujours perçu par une fraction non négligeable de la population comme le futur dirigeant du pays. Ce déficit politique dans l'approche de l'ONUSOM est sans doute typique d'une certaine naïveté américaine dans le traitement du problème somalien, ou d'une rancoeur très personnalisée du Secrétaire-général des Nations unies violemment pris à parti par Aydiid en janvier, lors de son rapide passage à Mogadiscio. Les conséquences d'une telle attitude sont désastreuses pour la recomposition d'un champ politique en Somalie, pour le travail humanitaire qui a pratiquement cessé dans la capitale, pour l'image des Nations unies en Somalie et les relations entre Somaliens et étrangers. Certes, il faut souligner que la situation est notoirement meilleure en dehors du sud de Mogadiscio, mais il est clair que l'ensemble du travail humanitaire pâtit cruellement du dilettantisme de l'ONUSOM. La logique humanitaire s'applique à penser les situations de crise suivant une grille d'analyse très simple, pour ne pas dire simpliste : urgence, réhabilitation, développement sont ses maitres-mots. La logique militaire, quant à elle, raisonne en fonction d'impératifs différents centrés sur la sécurité. Elles sont donc essentiellement différentes, ce qui n'implique pas qu'elles soient forcément contradictoires mais cela signifie qu'il faudra essayer de définir des règles d'interaction. Certains peuvent s'en émouvoir et déplorer ce qui peut apparaître comme une confusion, mais les évolutions actuelles et les formes présentes d'intervention internationale indiquent qu'elles vont devoir coexister. Les ONG, tout en soulignant les dangers d'une trop forte collusion de ces logiques, devraient réfléchir sur les implications de cette nouvelle donne. Dans le cas somalien, il y aurait eu deux niveaux d'articulation qui ont été occultés par tous les protagonistes extérieurs pour des raisons différentes : le désarmement des combattants et la recomposition d'un tissu social civil. Il est frappant de voir combien les critiques énoncées par les militaires ou les ONG, les uns à l'égard des autres, sont justifiées, et combien elles sont unilatérales. En caricaturant à peine, on retrouve implicitement deux modèles: celle de l'ONG héroïque, libérée des contraintes imposées en général par les Etats, qui ne s'interroge pas sur son rapport à la violence et sur son insertion dans l'économie de guerre et travaille dans l'illusion d'un accès immédiat aux populations; celle de l'armée de Liautey, qui assume une administration directe et le maintien de l'ordre, assurant un certain bien-être des populations. Il est frappant de constater que, dans ce débat sur l'humanitaire et le militaire, la question du devenir de la Somalie intervienne si peu. Pourtant le cas somalien est tout à fait paradigmatique. Aucun des termes du débat, tel qu'il est traditionnellement posé, n'est acceptable. Un certain type d'attitude critique 
vis-à-vis des Nations unies revient à abandonner la Somalie aux seigneurs de la guerre ; adhérer à l'ersatz d'analyse des Nations unies revient à privilégier une approche dont les résultats sont prévisibles : le renouveau d'un nationalisme xénophobe qui servira de ciment à un nouvel autoritarisme.

\section{NOTES}

1. Voir, pour une première réflexion, Cultures et conflits, $n^{\circ} 1$, et Marchal (R.), "Déplacements de population et conflits dans la Corne de l'Afrique", in SGDN, L'Afrique sub-saharienne : sécurité, stabilité et développement, Paris, La Documentation française, 1993.

2. A. de Waal, "Dix ans de famine dans la Corne de l'Afrique : un premier bilan de l'action humanitaire", Politique africaine, $\mathrm{n}^{\circ} 50$, juin 1993, p. 74.

3. Voir les deux ouvrages fondamentaux : Patman (R.), The Soviet Union in the Horn of Africa, Cambridge, Cambridge University Press, 1992 et Lefebvre (J.), Arms for the Horn : U.S. security policy in Ethiopia and Somalia 1953-1991, Pittsburgh, University of Pittsburgh Press, 1991.

4. Lewis (I.M.), A modern history of Somalia. Nation and State in the Horn of Africa, Boulder, Westview Press, 1988, pp. 246-248.

5. Lewis (I.M.), "The Ogaden and the fragility of the Somali segmentary nationalism", Africain Affairs, vol. 88, $\mathrm{n}^{\circ}$ 353, pp.573-580.

6. Voir Askin (S.), "Food aid diversion", Merip Report, vol. 17, n 2, mars-avril 1987, pp. 38-40.

7. Africa Watch, Somalia : A Government at war with its own people ; testimonies about the killings and the conflict in the North, Londres, Africa Watch, janvier 1990.

8. Sur l'ahurissante politique de l'administration américaine, consulter Lefebvre (J.), op. cit., p. 242-244. Heureusement le Congrès fut autrement plus lucide.

9. Compagnon (D.), "The Somali opposition fronts : some comments and questions", Horn of Africa, vol. 13, nº 1 \& 2, janvier-juin 1990, pp. 29-54.

10. Voitures $4 \times 4$, dont on a scié le toit et sur le plancher desquelles une plaque d'acier a été scellée afin d'y monter une mitrailleuse ou un canon sans recul.

11. Seule, l'organisation "SOS-enfants", qui gère un orphelinat à la sortie de Mogadiscio, maintiendra une présence permanente durant toute la crise.

12. Sur cette dimension de la violence, lire, Marchal (R.), "Formes de la violence dans un espace urbain en guerre : les mooryaan de Mogadiscio", Cahiers d'études africaines, n¹17, octobre 1993.

13. Les ONG ne sont pas les seules à être confrontées à ce problème : malheur au chercheur qui a des amis somaliens, il est lui aussi rapidement identifié selon leurs clans, sans hélas bénéficier de la même protection.

14. Marchal (R.) "La guerre à Mogadiscio", Politique Africaine, $n^{\circ} 46$, juin 1992, pp. 120-126.

15. Sur l'analyse de la gestion internationale de la crise somalienne, on peut se reporter à : Schraeder (P.), "La présence américaine dans la Corne de l'Afrique après la guerre 
froide : permanences et ruptures", Politique africaine, $\mathrm{n}^{\circ}$ 50, juin 1993, pp.59-73. et Marchal (R.), "Somalie : autopsie d'une intervention", Politique internationale, $\mathrm{n}^{\circ} 61$, octobre 1993.

16. Ce sont les populations Bantou (Sab), le plus souvent des agriculteurs, qui ont le plus souffert dans les zones de Baidoa et Bardhera.

17. Voir Marchal (R.), op. cit., (octobre 1993) pour une analyse des périodes UNITAF et ONUSOM.

18. African Rights, Somalia, Operation Restore Hope : a preliminary assessment, Londres, mai 1993.

19. Il faut quelques articles incisifs dans la presse internationale pour que l'armée américaine consente à laisser des bateaux transportant de l'aide accoster dans le port de Mogadiscio. On doit également mentionner le débat sur l'utilité de l'aide en décembre 1992 : voir A. de Waal, opus. cit., juin 1993 ou le rapport d'African Rights sur l'opération Restore Hope.

20. C'est la fameuse option "double zéro" : zéro blessé, zéro tué que les Américains appliqueront à leurs troupes jusqu'en juillet 1993, demandant aux autres contingents d'entreprendre les actions au sol les plus risquées...

21. African Rights, Somalia : Human rights abuses by the United Nations forces, Londres, juillet 1993.

\section{RÉSUMÉS}

"L'opinion publique internationale" a découvert, ou redécouvert le drame somalien à l'automne 92 aux vues des reportages soulignant les efforts des organisations humanitaires, et commentant l'intervention des forces de l'ONU à fin de la même année. Mais à se réjouir trop rapidement de ces interventions "salvatrices" on finit par oublier ou tout simplement occulter le rôle pour le moins ambigu joué par les organisations humanitaires, et l'action humanitaire en générale, en Somalie. La présence d'organisations humanitaires dans le pays n'est pas récente, loin s'en faut, puisqu'elles datent de la guerre avec l'Ethiopie. L'aide humanitaire a été associée à la stratégie politique et militaire du régime autoritaire et lui a servi de ressource d'appoint, par le détournement de l'aide notamment. L'aspect caractéristique de l'exemple somalien réside dans l'extrême complaisance des donateurs face au régime en place. A La chute du régime en 1991 le rôle joué par les ONG, peu nombreuses il est vrai, n'est pas moins ambigu, puisqu'elles ont contribué, certes involontairement, à l'économie politique du conflit. Enfin, l'intervention militaire onusienne et le retour massif d'une aide humanitaire excluant totalement la dimension politique, militaire et social, pour privilégier dans un premier temps uniquement la lutte contre la famine, n'a fait que fragiliser et détériorer la situation.

\section{INDEX}

Mots-clés : conflits, humanitaire, intervention militaire, Militaires

Index géographique : Corne de l'Afrique, Somalie

Index chronologique : 1990 - 2000 